\title{
On a general extending and constraining procedure for linear iterative methods
}

\author{
Aurelian Nicola, ${ }^{1}$, Stefania Petra ${ }^{2}$, Constantin Popa,${ }^{3}$ Christoph Schnörr ${ }^{4}$
}

\begin{abstract}
Algebraic Reconstruction Techniques (ART), on their both successive or simultaneous formulation, have been developed since early 70's as efficient "row action methods" for solving the image reconstruction problem in Computerized Tomography. In this respect, two important development directions were concerned with, firstly their extension to the inconsistent case of the reconstruction problem, and secondly with their combination with constraining strategies, imposed by the particularities of the reconstructed image. In the first part of our paper we introduce extending and constraining procedures for a general iterative method of ART type and we propose a set of sufficient assumptions that ensure the convergence of the corresponding algorithms. As an application of this approach, we prove that Cimmino's simultaneous reflections method satisfies this set of assumptions, and we derive extended and constrained versions for it. Numerical experiments with all these versions are presented on a head phantom widely used in the image reconstruction literature. We also considered hard thresholding constraining used in sparse approximation problems and applied it successfully to a $3 \mathrm{D}$ particle image reconstruction problem.
\end{abstract}

Keywords: Algebraic Reconstruction Techniques, inconsistent least squares problems, constraining strategies, Cimmino algorithm, Cimmino Extended algorithm, hard thresholding operator.

2000 MS Classification: 65F10, 65F20, 65F 22

\footnotetext{
${ }^{1}$ anicola@univ-ovidius.ro, Ovidius University Constanta, Faculty of Mathematics and Computer Science, Romania

${ }^{2}$ petra@math.uni-heidelberg.de, University of Heidelberg, Deptartment of Mathematics and Computer Science, Germany

${ }^{3}$ cpopa@univ-ovidius.ro, Ovidius University Constanta, Faculty of Mathematics and Computer Science, Romania; for this author the paper was partially supported by a DAAD Grant in the period 01.07 - 31.08.2009 at Institute for Computer Science 10 (Systemsimulation), Friedrich-Alexander-Universität Erlangen-Nürnberg, Germany

${ }^{4}$ schnoerr@math.uni-heidelberg.de, University of Heidelberg, Deptartment of Mathematics and Computer Science, Germany
} 


\section{Introduction}

Many classes of "real world problems" give rise, after appropriate discretizations to big, sparse and ill-conditioned linear systems of equations of the form $A x=b$, where the $m \times n$ matrix $A$ contains information concerning the problem, whereas $b \in \mathbb{R}^{m}$ represents measured "effects" produced by the unknown "cause" $x \in \mathbb{R}^{n}$. But, due to inevitable measurements errors, the "effect" $b$ may go out of the "range of action" of the problem information matrix $A$, such that the above system of equations becomes inconsistent and must be reformulated in the least squares sense: find $x \in \mathbb{R}^{n}$ such that

$$
\|A x-b\|=\min \left\{\|A z-b\|, z \in \mathbb{R}^{n}\right\}
$$

where $\|\cdot\|$ is the Euclidean norm $(\langle\cdot, \cdot\rangle$ will be the corresponding scalar product) on some space $\mathbb{R}^{q}$.

Remark 1 Concerning the matrix involved in (1) we shall suppose for the whole paper that it has nonzero rows $A_{i}$ and columns $A^{j}$, i.e.

$$
A_{i} \neq 0, i=1, \ldots, m, A^{j} \neq 0, j=1, \ldots, n
$$

These assumptions are not essential restrictions of the generality of the problem (1) because, if $A$ has null rows and/or columns, it can be easily proved that they can be eliminated without affecting its set of classical $(S(A ; b)$, in the consistent case) or least squares ( $L S S(A ; b)$, in the inconsistent case) solutions.

As a very important example of such problems (to which also refer the numerical experiments in the last section of our paper) is the Image Reconstruction from Projections in Computerized Tomography. Its algebraic mathematical model, although essentially based on an integral equation formulation gives rise after the "rays $\times$ pixels" discretization procedure (see for details [16], [6]) to least squares problems of the form (1). For numerical solution of these problems the class of Algebraic Reconstruction Techniques (ART) were very much developed in the last 40 years (see [6] and references therein). These methods are iterative "row-action" algorithms (i.e. they use rows or blocks

of rows of the system matrix $A$ in each iteration, without changing the values of its entries or its structure; see [6]) and are "classified" according to the way in which the rows/blocks of rows are "visited" in each iteration: 
(I) successive ART, having as standard method Kaczmarz's algorithm [19]

(II) simultaneous ART, having as standard method Cimmino's algorithm [10]

According to these standard algorithms, we shall consider in our paper ARTlike methods of the following general form.

Algorithm General ART (GenART). Initialization: $x^{0} \in \mathbb{R}^{n}$

Iterative step:

$$
x^{k+1}=T x^{k}+R b,
$$

where $T: n \times n$ and $R: n \times m$ are real matrices.

Remark 2 We introduce the following assumption on the matrices $T$ and $R$ : they have an explicit expression in terms of the rows of $A$ and components of the right hand side of (1), i.e. if we change $A$ and $b$ to $\bar{A}$ and $\bar{b}$ we can define in a similar way as in (3) an iterative process of the form $x^{k+1}=$ $\bar{T} x^{k}+\bar{R} \bar{b}$. Examples in this sense are given by the projection algorithms appearing in image reconstruction from projections: Kaczmarz, Cimmino, Landweber, DW, SART, etc (see e.g. [6, 7, 8, 16, 13, 18, 20, 21, 31] and references therein).

Almost all of these algorithms generate sequences convergent to a solution of the problem (1) in the consistent case, whereas in the inconsistent one the sequence $\left(x^{k}\right)_{k \geq 0}$ still converges, but the limit is not any more an element of $L S S(A ; b)$. In this respect have been designed extensions of the algorithm (3) for the inconsistent case of (1) which are based on relaxation parameters, column relaxations or supplementary steps introduced in the iteration (see $[21,11,23,26,2,30]$ and references therein). Moreover, for problems related to image reconstruction in computerized tomography the iteration step (3) was combined with a constraining strategy, usually acting on the components of the successive approximations $x^{k}=\left(x_{1}^{k}, \ldots, x_{n}^{k}\right)^{T}$ (see $[20,23,28]$ ).

In this paper we will analyse from these points of view the General ART algorithm (3). The paper is organised as follows: in section 2 we present the essential assumptions on the matrices $T$ and $R$ from (3) which ensure both the possibility to extend it to inconsistent problems and to combine it with a class of constraining strategies. Moreover, we prove that these assumptions are sufficient for obtaining convergence results for the extended and constrained versions of the General ART method. As an application of 
these main results of the paper, in section 3 we prove that Cimmino's reflections algorithm ([10]) satisfies all the above assumptions and we derive its extended and constrained versions. Even though Cimmino's method was created many years ago, it can be now view as a special case of Landweber method, such that the Constrained Cimmino algorithm can be retrieved as a particular" projected Landweber method" (see e.g. [1]) or as a "gradient projection" algorithm (see [15]). The fact that it was used in this section as an application for our considerations in section 2 is only an historical poin of view.

Regarding the extension procedure proposed in (35)-(37), it differs from the older "multi-steps" methods (see [30, 2]) or methods that uses in the inconsistent case for (1), the associated augmented system (which is always consistent) by the following two aspects: firstly, the modification of the right-hand side in (36) is included in the iteration of the extended algorithm, thus in the global convergence of the algorithm (so do no more appear accumulation of errors due to approximate solutions in the different steps of "multi-steps" methods), and the second one, the fact that, acting on the initial problem, the extended method (35)-(37) is influenced by its condition number and not by the squared one, as in the case of augmented system or normal equa-

tion (see [3] and the numerical experiments in [22]). Moreover, we want to point out that the extending and constraining approach developed under the assumptions (8)-(12) is quite general and can include other algorithms, in image reconstruction or elsewhere (e.g. DW or SART algorithms; see e.g. [18]), for which it will be possible to prove these assumptions. Moreover, once these assumptions are verified, we get already three new algorithms: extended, constrained and constrained extended.

The last section of the paper is devoted to experiments with all these versions on a phantom widely used in the literature. Moreover, we considered different constraining strategies, including hard thresholding, and compared these in the context of particle image reconstruction.

\section{The general extending and constraining procedures}

We shall first introduce some notations. The spectrum and spectral radius of a square matrix will be denoted by $\sigma(B)$ and $\rho(B)$, respectively. By 
$A^{T}, \mathcal{N}(A), \mathcal{R}(A)$ we shall denote the transpose, null space and range of $A$. $P_{S}(x)$ will be the orthogonal (Euclidean) projection onto a vector subspace $S$ of some $\mathbb{R}^{q} . S(A ; b), L S S(A ; b)$ will stand for the set of classical or leastsquares solutions of (1), respectively. By $x_{L S}$ we will denote the (unique) solution with minimal Euclidean norm (in both cases). In the general case for (1) the following properties are known:

$$
\begin{gathered}
b=P_{\mathcal{R}(A)}(b)+P_{\mathcal{N}\left(A^{T}\right)}(b), \\
L S S(A ; b)=x_{L S}+\mathcal{N}(A) \text { and } x \in L S S(A ; b) \Leftrightarrow A x=P_{\mathcal{R}(A)}(b), \\
S(A ; b)=x_{L S}+\mathcal{N}(A) \text { and } x \in S(A ; b) \Leftrightarrow A x=b .
\end{gathered}
$$

Moreover, $x_{L S}$ is the unique element of $L S S(A ; b)$ (or $S(A ; b)$ ) which belongs to the subspace $\mathcal{R}\left(A^{T}\right)$. The spectral norm of $A$ will be defined by

$$
\|A\|=\sup _{x \neq 0} \frac{\|A x\|}{\|x\|}=\sup _{\|x\|=1}\|A x\| .
$$

Now, we introduce the following basic assumptions on the above considered matrices $T$ and $R$.

$$
\begin{gathered}
I-T=R A . \\
\text { if } x \in \mathcal{N}(A) \text { then } T x=x \in \mathcal{N}(A) . \\
\text { if } x \in \mathcal{R}\left(A^{T}\right) \text { then } T x \in \mathcal{R}\left(A^{T}\right) . \\
\forall y \in \mathbb{R}^{m}, R y \in \mathcal{R}\left(A^{T}\right) . \\
\text { if } \tilde{T}=T P_{\mathcal{R}\left(A^{T}\right)} \text { then }\|\tilde{T}\|<1 .
\end{gathered}
$$

Proposition 1 If (8) - (12) hold then the following are true. (i) $I-\tilde{T}$ is invertible and the $n \times m$ matrix $G$ defined by

$$
G=(I-\tilde{T})^{-1} R
$$

satisfies

$$
A G A=A \text { and } G P_{\mathcal{R}(A)}(b)=x_{L S} .
$$

(ii) The matrix $T$ has the properties

$$
\|T x\|=\|x\| \text { if and only if } x \in \mathcal{N}(A)
$$


and

$$
\|T\| \leq 1 \text {. }
$$

(iii) For the approximations $x^{k}, k \geq 0$ generated with the algorithm (3) we have

$$
P_{\mathcal{N}(A)}\left(x^{k}\right)=P_{\mathcal{N}(A)}\left(x^{0}\right), \quad \forall k \geq 0 .
$$

Proof. (i) From (12) (see e.g. [3]) it results that the matrix $I-\tilde{T}$ is invertible and

$$
(I-\tilde{T})^{-1}=\sum_{i \geq 0} \tilde{T}^{i}
$$

From the definition of $\tilde{T}$ in (12) and (9) (see also [31]) we get

$$
T=P_{\mathcal{N}(A)}+\tilde{T}, \tilde{T} P_{\mathcal{N}(A)}=P_{\mathcal{N}(A)} \tilde{T}=0 .
$$

Then, from (8), (13), (19) and (18) we successively obtain

$$
\begin{gathered}
A G A=A(I-\tilde{T})^{-1} R A=A(I-\tilde{T})^{-1}(I-T)= \\
A(I-\tilde{T})^{-1}\left((I-\tilde{T})-P_{\mathcal{N}(A)}\right)=A-A(I-\tilde{T})^{-1} P_{\mathcal{N}(A)}=A,
\end{gathered}
$$

i.e. the first equality in (14). Then, because $P_{\mathcal{R}(A)}(b) \in \mathcal{R}(A)$ we get from the first equality in (14) $A G P_{\mathcal{R}(A)}(b)=P_{\mathcal{R}(A)}(b)$, which means that $x^{*}=$ $G P_{\mathcal{R}(A)}(b) \in L S S(A ; b)$ (see e.g. [3]). But, from (11), (18) the definition of $\tilde{T}$ it results that $x^{*} \in \mathcal{R}\left(A^{T}\right)$, i.e. $x^{*}=x_{L S}$, thus by the unicity of $x_{L S}$ which proves the second equality in (14).

(ii) The "if" part results directly from (9). For the "only if" one, let $x \in \mathbb{R}^{n}$ be such that $\|T x\|=\|x\|$ holds. Then, if $x=x^{\prime}+x^{\prime \prime}=P_{\mathcal{N}(A)}(x)+P_{\mathcal{R}\left(A^{T}\right)}(x)$, and $x^{\prime \prime} \neq 0$, from (9) and (10) we get $T x^{\prime} \in \mathcal{N}(A)$ and $T x^{\prime \prime} \in \mathcal{R}\left(A^{T}\right)$. Thus, by also using (12) we successively obtain

$$
\begin{gathered}
\|T x\|^{2}=\left\|T x^{\prime}\right\|^{2}+\left\|T x^{\prime \prime}\right\|^{2} \leq\left\|x^{\prime}\right\|^{2}+\|\tilde{T}\|^{2} \quad\left\|x^{\prime \prime}\right\|^{2} \\
<\left\|x^{\prime}\right\|^{2}+\left\|x^{\prime \prime}\right\|^{2}=\|x\|^{2}
\end{gathered}
$$

which contradicts our initial assumption about the vector $x$. It follows that $x^{\prime \prime}=0$, i.e. $x \in \mathcal{N}(A)$. The inequality (16) results from (20) for an arbitrary $x \in \mathbb{R}$ (in which case the last inequality is not any more strict). 
(iii) We will use the mathematical induction. Let us suppose that $k \geq 0$ is such that (17) holds. For $k+1$ we have, by also using (3) and (19)

$$
x^{k+1}=T x^{k}+R b=P_{\mathcal{N}(A)}\left(x^{k}\right)+\tilde{T} x^{k}+R b .
$$

But, from (10) and (11) we obtain that $\tilde{T} x^{k}+R b \in \mathcal{R}\left(A^{T}\right)$, i.e. $P_{\mathcal{N}(A)}\left(x^{k+1}\right)=$ $P_{\mathcal{N}(A)}\left(x^{k}\right)=P_{\mathcal{N}(A)}\left(x^{0}\right)$ which completes the proof.

The convergence properties of the algorithm GenART (3) are given in the following result.

Theorem 1 Let us suppose that the matrices $T$ and $R$ satisfy the assumptions (8)-(12). Then, for any $x^{0} \in \mathbb{R}^{n}$ the sequence $\left(x^{k}\right)_{k \geq 0}$ generated with the algorithm (3) converges and

$$
\lim _{k \rightarrow \infty} x^{k}=P_{\mathcal{N}(A)}\left(x^{0}\right)+G b .
$$

If the problem (1) is consistent, then

$$
G b=x_{L S} \text { and } \lim _{k \rightarrow \infty} x^{k}=P_{\mathcal{N}(A)}\left(x^{0}\right)+x_{L S} \in S(A ; b) .
$$

Proof. Let $e^{k}=x^{k}-\left(P_{\mathcal{N}(A)}\left(x^{0}\right)+G b\right)$ be the error vector at iteration $k$ (see (21)). Using (3), (13), (19) and (17) we successively obtain

$$
\begin{gathered}
e^{k}=x^{k}-\left(P_{\mathcal{N}(A)}\left(x^{0}\right)+G b\right)=T x^{k-1}+R b-\left[P_{\mathcal{N}(A)}\left(x^{0}\right)+[(I-\tilde{T})+\tilde{T}](I-\tilde{T})^{-1} R b\right]= \\
\tilde{T} x^{k-1}-\tilde{T}(I-\tilde{T})^{-1} R b=\tilde{T}\left(x^{k-1}-P_{\mathcal{N}(A)}\left(x^{0}\right)-G b\right)=\tilde{T} e^{k-1},
\end{gathered}
$$

i.e., by a recursive argument

$$
e^{k}=\tilde{T}^{k} e^{0}, \forall k \geq 0
$$

But, according to $(12)$ we get that $\lim _{k \rightarrow \infty} e^{k}=0$, from which we get $(21)$. The second part of the theorem (22) results from Proposition 1 (i).

Theorem 2 Let $x^{*}$ be the limit point in (21). Then we have the a priori estimate

$$
\left\|x^{k}-x^{*}\right\| \leq \frac{\kappa^{k}}{1-\kappa}\left\|x^{0}-x^{1}\right\|
$$


and the a posteriori estimate

$$
\left\|x^{k+1}-x^{*}\right\| \leq \frac{\kappa}{1-\kappa}\left\|x^{k+1}-x^{k}\right\|,
$$

where $\kappa=\|\widetilde{T}\|$. In particular, the convergence rate of sequence $\left\{x^{k}\right\}_{k}$ is linear.

Proof. Let $\left\{x^{k}\right\}_{k}$ be the sequence generated by GenART for an arbitrary initial approximation $x^{0} \in \mathbb{R}^{n}$ and suppose that the matrices $T$ and $R$ satisfy the assumptions (8)-(12). Then using (17) we can rewrite equation (3) as

$$
x^{k+1}=\widetilde{T} x^{k}+P_{\mathcal{N}(A)}\left(x^{0}\right)+R b=: F\left(x^{k}\right),
$$

since we can decompose $T$ according to (19). The mapping $F$ is a contraction with Lipschitz constant $\kappa:=\|\widetilde{T}\|$. Banach's fixed-point theorem asserts additionally to the convergence of sequence $\left\{x^{k}\right\}_{k}$ to a fixed point of $F$, the estimates in (24) and (25).

Remark 3 We claim that the above set of sufficient assumptions (8)-(12) are also necessary to obtain the results in proposition 1 and Theorem 1, but we don't have yet a rigorous proof of this statement.

According to Remark 2 let $U$ and $S$ be the $m \times m$, respectively $m \times n$ matrices, similar to $T$ and $R$ from (3), respectively but for the (always consistent) system

$$
A^{T} y=0 .
$$

Then, the corresponding algorithm of the form (3) with $U$ and $S$ will be written as

$$
y^{k+1}=U y^{k}+S \cdot 0=U y^{k}, \forall k \geq 0,
$$

with $y^{0} \in \mathbb{R}^{m}$ the initial approximation. Our general assumptions (8) - (12) and Proposition 1 will assign the following properties to the matrix $U$

$$
U\left(\mathcal{N}\left(A^{T}\right)\right) \subset \mathcal{N}\left(A^{T}\right), \quad U(\mathcal{R}(A)) \subset \mathcal{R}(A),
$$

$$
\text { if } \tilde{U}=U P_{\mathcal{R}(A)} \text { then } U=P_{\mathcal{N}\left(A^{T}\right)} \oplus \tilde{U} \text { and } P_{\mathcal{N}\left(A^{T}\right)} \tilde{U}=\tilde{U} P_{\mathcal{N}\left(A^{T}\right)}=0 \text {, }
$$

$$
U^{k}=P_{\mathcal{N}\left(A^{T}\right)} \oplus \tilde{U}^{k}, \quad\|\tilde{U}\|<1 \text { and } P_{\mathcal{N}\left(A^{T}\right)}\left(y^{k}\right)=P_{\mathcal{N}\left(A^{T}\right)}\left(y^{0}\right), \forall k \geq 0 .
$$

Moreover, according to the above Theorem 1 the following convergence result will hold for the algorithm (28). 
Theorem 3 For any $y^{0} \in \mathbb{R}^{m}$, the sequence $\left(y^{k}\right)_{k \geq 0}$ generated with the algorithm (28) converges and

$$
\lim _{k \rightarrow \infty} y^{k}=P_{\mathcal{N}\left(A^{T}\right)}\left(y^{0}\right) .
$$

Proof. Let

$$
\varepsilon^{k}=y^{k}-P_{\mathcal{N}\left(A^{T}\right)}\left(y^{0}\right)
$$

be the error vector at iteration $k$ (see (32)). Using (28), (30), (31) and (33) we successively obtain

$$
\begin{gathered}
\varepsilon^{k}=U y^{k-1}-P_{\mathcal{N}\left(A^{T}\right)}\left(y^{0}\right)=\tilde{U} y^{k-1}+P_{\mathcal{N}\left(A^{T}\right)}\left(y^{k-1}\right)-P_{\mathcal{N}\left(A^{T}\right)}\left(y^{0}\right)=\tilde{U} y^{k-1}= \\
\tilde{U}\left(y^{k-1}-P_{\mathcal{N}\left(A^{T}\right)}\left(y^{0}\right)\right)=\tilde{U} e^{k-1} .
\end{gathered}
$$

But, from (31) we get $\lim _{k \rightarrow \infty} \varepsilon^{k}=0$ from which (32) holds and completes the proof.

If $y^{0}=b$, from (32) we would get $\lim _{k \rightarrow \infty} y^{k}=P_{\mathcal{N}\left(A^{T}\right)}(b)$, thus $\lim _{k \rightarrow \infty}(b-$ $\left.y^{k}\right)=P_{\mathcal{R}(A)}(b)$. This simple observation allows us to consider the following extension of the general algorithm GenART.

Algorithm Extended General ART (EGenART).

Initialization: $x^{0} \in \mathbb{R}^{n}, y^{0}=b$

Iterative step:

$$
\begin{aligned}
y^{k+1} & =U y^{k}, \\
b^{k+1} & =b-y^{k+1}, \\
x^{k+1} & =T x^{k}+R b^{k+1} .
\end{aligned}
$$

Theorem 4 Let us suppose that the matrices $T$ and $R$ satisfy (8)-(12) and the assumption from Remark 2. Then, for any $x^{0} \in \mathbb{R}^{n}$, the sequence $\left(x^{k}\right)_{k \geq 0}$ generated with the algorithm (35) - (37) converges and

$$
\lim _{k \rightarrow \infty} x^{k}=P_{\mathcal{N}(A)}\left(x^{0}\right)+x_{L S} \in L S S(A ; b) .
$$

Proof. Let $e^{k}=x^{k}-\left(P_{\mathcal{N}(A)}\left(x^{0}\right)+x_{L S}\right)$ be the error vector at iteration $k$ (see (38)). Using (37), (13), (14), (19) and (17) we successively obtain

$$
\begin{gathered}
e^{k}=x^{k}-\left(P_{\mathcal{N}(A)}\left(x^{0}\right)+x_{L S}\right)=T x^{k-1}+R b^{k}- \\
{\left[P_{\mathcal{N}(A)}\left(x^{0}\right)+[(I-\tilde{T})+\tilde{T}](I-\tilde{T})^{-1} R P_{\mathcal{R}(A)}(b)\right]=}
\end{gathered}
$$




$$
\begin{gathered}
\tilde{T} x^{k-1}+R b^{k}-R P_{\mathcal{R}(A)}(b)-G P_{\mathcal{R}(A)}(b)= \\
\tilde{T}\left(x^{k-1}-P_{\mathcal{N}(A)}\left(x^{0}\right)-x_{L S}+R\left(b-y^{k}-P_{\mathcal{R}(A)}(b)\right) .\right.
\end{gathered}
$$

Because $y^{0}=b$, from (28) and (4) we get

$$
y^{k}=U y^{k-1}=\tilde{U} y^{k-1}+P_{\mathcal{N}\left(A^{T}\right)}(b), \forall k \geq 0 .
$$

From (40) we obtain for the second term in (39)

$$
R\left(b-y^{k}-P_{\mathcal{R}(A)}(b)\right)=-R \tilde{U} y^{k-1},
$$

which gives us by a recursive argument

$$
R\left(b-y^{k}-P_{\mathcal{R}(A)}(b)\right)=-R \tilde{U}^{k} y^{0}=-R \tilde{U}^{k} b .
$$

From (39), (41) and again a recursive argument we get

$$
e^{k}=\tilde{T} e^{k-1}-R \tilde{U}^{k} b=\tilde{T}^{k} x^{0}-R \tilde{U}^{k} b .
$$

From (42), (12) and (31) we obtain that $\lim _{k \rightarrow \infty} e^{k}=0$, from which (38) holds and completes the proof.

Remark 4 A different extension procedure has been proposed in [12]. It uses similar ideas in the convergence proof, but under different initial assumptions.

By using the decomposition (4) and the second equality in (14) we obtain that the limit $(21)$ of the sequence $\left(x^{k}\right)_{k \geq 0}$ generated by (3) can be written as

$$
\lim _{k \rightarrow \infty} x^{k}=P_{\mathcal{N}(A)}\left(x^{0}\right)+x_{L S}+\Delta, \text { with } \Delta=(I-\tilde{Q})^{-1} R P_{\mathcal{N}\left(A^{T}\right)}(b) .
$$

In the paper $[20]$ the authors consider a constraining function, $C: \mathbb{R}^{n} \longrightarrow$ $\mathbb{R}^{n}$ with a closed image $\operatorname{Im}(C) \subset \mathbb{R}^{n}$ and the properties

$$
\begin{gathered}
\|C x-C y\| \leq\|x-y\|, \\
\text { if }\|C x-C y\|=\|x-y\| \text { then } C x-C y=x-y, \\
\text { if } y \in \operatorname{Im}(C) \text { then } y=C y .
\end{gathered}
$$


Example 1 "Box constraining" function

$$
(C x)_{i}= \begin{cases}x_{i}, & x_{i} \in\left[\alpha_{i}, \beta_{i}\right] \\ 0, & x_{i}<\alpha_{i} \\ 1, & x_{i}>\beta_{i}\end{cases}
$$

i.e. $C$ is the orthogonal projection onto the closed convex set $V=\left[\alpha_{1}, \beta_{1}\right] \times$ $\cdots \times\left[\alpha_{n}, \beta_{n}\right] \subset \mathbb{R}^{n}$, for which it is well known that (44)-(46) hold (see e.g. [17]) and its image is closed $(\operatorname{Im}(C)=V)$.

Example 2 The hard thresholding operator from [4], which has the following general form $(\alpha \geq 0)$

$$
H_{\alpha}(y)=\left(h_{\alpha}\left(y_{1}\right), \ldots, h_{\alpha}\left(y_{n}\right)\right), y=\left(y_{1}, \ldots, y_{n}\right) \in \mathbb{R}^{n}
$$

with

$$
h_{\alpha}\left(y_{i}\right)=\left\{\begin{array}{cc}
0, & \left|y_{i}\right|<\alpha \\
y_{i}, & y_{i} \in(-\infty,-\alpha] \cup[\alpha, \infty)
\end{array}, i=1, \ldots, n .\right.
$$

From the definition (48)-(49) it results that $\operatorname{Im}\left(H_{\alpha}\right) \subset \mathbb{R}^{n}$ is closed. Moreover, it satisfies (44)-(46). Indeed, we firstly have to observe that, by direct computations and using (48)-(49) we obtain

$$
\left|h_{\alpha}\left(x_{i}\right)-h_{\alpha}\left(y_{i}\right)\right| \leq\left|x_{i}-y_{i}\right| \text { and } h_{\alpha}\left(h_{\alpha}\left(x_{i}\right)\right)=h_{\alpha}\left(x_{i}\right), \forall x_{i}, y_{i} \in \mathbb{R} .
$$

Then (44) and (46) hold directly from (50). For (45) we observe that if we have the equality of norms in (44) then $\sum_{i=1}^{n}\left(h_{\alpha}\left(x_{i}\right)-h_{\alpha}\left(y_{i}\right)\right)^{2}=\sum_{i=1}^{n}\left(x_{i}-\right.$ $\left.y_{i}\right)^{2}$ which combined with (50) gives us $\left(h_{\alpha}\left(x_{i}\right)-h_{\alpha}\left(y_{i}\right)\right)^{2}=\left(x_{i}-y_{i}\right)^{2}, \forall i=$ $1, \ldots, n$, i.e. $(45)$.

\section{Algorithm Constrained General ART (CGenART).}

Initialization: $x^{0} \in \mathbb{R}^{n}$

Iterative step:

$$
x^{k+1}=C\left[T x^{k}+R b\right] .
$$

Theorem 5 Let us suppose that the the matrices $T$ and $R$ satisfy (8)-(12), the constraining function $C$ satisfies (44) - (46) and the set $\mathcal{V}^{*}$, defined by

$$
\mathcal{V}^{*}=\{y \in \operatorname{Im}(C), y-\Delta \in L S S(A ; b)\}
$$

is nonempty. Then, for any $x^{0} \in \operatorname{Im}(C)$ the sequence $\left(x^{k}\right)_{k \geq 0}$ generated by the algorithm CGenART converges and its limit belongs to the set $\mathcal{V}^{*}$. 
Proof. We follow exactly the proof in [20] . In this respect, the proof of Lemma 6[20] holds from (43), (9), (3), (21), (46), (95)[20], (44), (16), (45), (9) and (12). The proof of Theorem 7 then holds from (3) and the above Lemma 6[20].

Remark 5 If the problem (1) is consistent then

$$
\Delta=0 \text { and } \mathcal{V}^{*}=S(A ; b) \cap \operatorname{Im}(C),
$$

i.e., the algorithm CGenART generates a "constrained" solution of (1).

Remark 6 We have to observe that all the assumptions (44) - (46) are necessary in the proof of the above Theorem 5.

Algorithm Constrained Extended General ART (CEGenART). Initialization: $x^{0} \in \operatorname{Im}(C), y^{0}=b$;

Iterative step:

$$
\begin{aligned}
y^{k+1} & =U\left(y^{k}\right), \\
b^{k+1} & =b-y^{k+1}, \\
x^{k+1} & =C\left[T x^{k}+R b^{k+1}\right],
\end{aligned}
$$

with $U, T, R$ from (28), (3) and $C$ as in (44)-(46). We shall suppose that at least one least squares solution exists in $\operatorname{Im}(C)$ (see the hypothesis of Theorem 5), i.e. the set $\mathcal{V}$ defined below is nonempty

$$
\mathcal{V}=L S S(A ; b) \cap \operatorname{Im}(C) \neq \emptyset \text {. }
$$

Theorem 6 Let us suppose that the the matrices $T$ and $R$ satisfy (8)-(12), the constraining function $C$ satisfies (44) and (46) and the set $\mathcal{V}$ satisfies (57). Then, for any $x^{0} \in \operatorname{Im}(C)$, the sequence $\left(x^{k}\right)_{k \geq 0}$ generated with the algorithm CEGenART (54) - (56) converges to an element of $\mathcal{V}$.

Proof. We follow exactly the proof in [28] . In this respect we have: for the proof of Lemma 1 we need (54) and (30); for the proof of Lemma 2 we need (12), (16), (32) and (19); for the proof of Lemma 3 we need (55), the above Lemma 1, (32), (56), the above Lemma 2, (44), (46), (16), (12), (9) and (15); for the proof of Lemma 4 we need the above Lemma 3, (57), (44), (55), (32), (16) and the already mentioned lemmas. Finally, Lemma 5 holds 
from the above mentioned results.

Remark 7 The assumption that the set $\mathcal{V}$ from (57) is nonempty is directly connected with the (level of) perturbation of $b$ in (1), which makes it inconsistent. It requests that we still have least squares solutions in $\operatorname{Im}(C)$.

A projection method that fits into the above considerations is Kaczmarz's successive projection algorithm from [19]. The properties (8)-(12) were proved in the paper [31]. But, independently on the general approach presented in this section, a theorem of the form Theorem 1 was proved in [31], the extension of the form (35)-(37) was first proposed in [25], a constrained version of Kaczmarz method in [20] and a constrained version of Kaczmarz Extended method in [28]. In the next section of the paper we shall obtain all these versions for Cimmino's algorithm, by simply proving that it satisfies assumptions (8)-(12) and then applying the above constructions.

\section{Application - Cimmino's reflections algorithm}

In the paper [10], Cimmino considers a consistent problem of the form (1) where $A$ is an $m \times n$ real matrix and $b \in \mathbb{R}^{n}$. A solution point will lie in the intersection of the $m$ hyperplanes described by

$$
H_{i}:=\left\{x \mid A_{i}^{T} x=b_{i}\right\}, \quad i=1, \ldots, m .
$$

Given a current approximation $x^{k}$, the next one $x^{k+1}$ is constructed as

$$
x^{k+1}=\sum_{i=1}^{m} \frac{\omega_{i}}{\omega} y^{k, i}
$$

where $y^{k, i}$ are the reflections of $x^{k}$ with respect to the hyperplane (58), defined by

$$
y^{k, i}=x^{k}+2 \frac{b_{i}-A_{i}^{T} x^{k}}{\left\|A_{i}\right\|^{2}} A_{i} \text { and } \omega_{i}>0, \quad \omega=\sum_{i=1}^{m} \omega_{i} .
$$

From (59) and (60) we derive for $T, R$ in (3) the following expressions

$$
T=\sum_{i=1}^{m} \frac{\omega_{i}}{\omega} S_{i}, \quad S_{i}:=I-2 \frac{A_{i} A_{i}^{T}}{\left\|A_{i}\right\|^{2}}, \quad R=\sum_{i=1}^{m} \frac{\omega_{i}}{\omega} \frac{b_{i}}{\left\|A_{i}\right\|^{2}} A_{i} .
$$


Then, Cimmino's algorithm (59) can be written as follows.

Algorithm Cimmino (Cmm). Initialization: $\omega_{i}>0, i=1, \ldots, m ; x^{0} \in$ $\mathbb{R}^{n}$

Iterative step:

$$
x^{k+1}=T x^{k}+R b .
$$

\section{Proposition 2 If}

$$
\operatorname{rank}(A) \geq 2,
$$

then the matrices $T$ and $R$ from (61) satisfies the assumptions (8)-(12).

Proof. The statements in (8) - (11) follow directly from (61) and the fact that $\mathcal{R}\left(A^{T}\right)=s p\left\{A_{1}, \ldots, A_{m}\right\}$. For (12) we first observe that the orthogonal reflectors $S_{i}$ from (61) are also isometric transformations, thus

$$
\left\|S_{i} x\right\|=\|x\|, \forall x \in \mathbb{R}^{n} \text { and }\left\|S_{i}\right\|=1, \forall i=1, \ldots, m .
$$

Then, for an arbitrary $x \in \mathbb{R}^{n}$ we get from (60) and (64)

$$
\|T x\|=\left\|\sum_{i=1}^{m} \frac{\omega_{i}}{\omega} S_{i} x\right\| \leq \sum_{i=1}^{m} \frac{\omega_{i}}{\omega}\left\|S_{i} x\right\|=\|x\|,
$$

which together with (9) give us $\|T\|=1$, thus

$$
\|\tilde{T}\| \leq 1
$$

Let us now suppose that we have equality in (66) and let $x \in \mathcal{R}\left(A^{T}\right), x \neq 0$ be such that (see also (65))

$$
\|\tilde{T}(x)\|=\|T(x)\|=\left\|\sum_{i=1}^{m} \frac{\omega_{i}}{\omega} S_{i} x\right\|=\sum_{i=1}^{m} \frac{\omega_{i}}{\omega}\left\|S_{i} x\right\|=\|x\| .
$$

By the nonsingularity of $S_{i}$ we have $S_{i} x \neq 0$ for all $i=1, \ldots, m$. Since the Euclidean norm is strictly convex, $\omega_{i}>0$ and $\sum_{i=1}^{m} \frac{\omega_{i}}{\omega}=1$ the equality from (67) only holds if $S_{1} x=\cdots=S_{m} x$. Let us suppose that $S_{1} x=S_{i} x$ for all $i=2, \ldots, m$. This is equivalent to

$$
\frac{A_{1}^{T} x}{\left\|A_{1}\right\|^{2}} A_{1}-\frac{A_{i}^{T} x}{\left\|A_{i}\right\|^{2}} A_{i}=0 \text { for all } i=2, \ldots, m .
$$

Since we have the assumption (63) on $A$, the equalities above imply that $A_{i}^{T} x=0, \forall i=1, \ldots, m$, i.e. $x \in \mathcal{N}(A)$ thus $x=0$ which contradicts the 
initial assumption on it. Thus (12) holds and the proof is complete.

According to the results from section 2 we can now first design the Extended Cimmino algorithm following the general formulation (35)-(37). According to (61) and (27), the matrix $U$ from (28) will be given by

$$
U=\sum_{i=1}^{n} \frac{\alpha_{j}}{\alpha} F_{j}, \text { with } F_{j}=I-2 \frac{A^{j} A^{j^{T}}}{\left\|A^{j}\right\|^{2}}, \text { and } \alpha=\sum_{j=1}^{n} \alpha_{j},
$$

and $\alpha_{j}>0$ arbitrary weights.

Algorithm Extended Cimmino (ECmm).

Initialization: $\omega_{i}>0, i=1, \ldots, m ; \alpha_{j}>0, j=1, \ldots, n, x^{0} \in \mathbb{R}^{n}, y^{0}=b$

Iterative step:

$$
\begin{aligned}
y^{k+1} & =U y^{k}, \\
b^{k+1} & =b-y^{k+1}, \\
x^{k+1} & =T x^{k}+R b^{k+1} .
\end{aligned}
$$

The corresponding constrained versions, $\mathbf{C C m m}$ and $\mathbf{C E C m m}$ are directly

derived from (62) and (69)-(71) following the general formulations (51) and (54)-(56), respectively.

\section{$4 \quad$ Numerical experiments}

The experiments from this first part of the section do not intend to compare Cimmino-like algorithms designed in section 3 with others more efficient ones (see also the comments on section 1). We wanted only to show by some examples the importance of such kind of developments: extension and constraining. In our first set of experiments we used the head phantom from the paper [9] $(63 \times 63$ pixels resolution with the scanning matrix with 1376 rays - i.e. $m$, the number of rows in $A$ ). A consistent and an inconsistent right hand side $b$ was used in our reconstruction experiments, together with the following measures for the approximation errors (see also [16]).

- $x^{e x}=$ head phantom ; $n=63^{2}=3969$

- $x^{e x}=\left(x_{1}^{e x}, \ldots, x_{n}^{e x}\right)^{T} ; x^{k}=\left(x_{1}^{k}, \ldots, x_{n}^{k}\right)^{T} ; \bar{x}^{e x}=\frac{\sum_{i=1}^{n} x_{i}^{e x}}{n} ; \bar{x}^{k}=\frac{\sum_{i=1}^{n} x_{i}^{k}}{n}$

- Distance $=\sqrt{\frac{\sum_{i=1}^{n}\left(x_{i}^{e x}-x_{i}^{k}\right)^{2}}{\sum_{i=1}^{n}\left(x_{i}^{e x}-\bar{x}^{e x}\right)^{2}}}$ 
- Relative error $=\frac{\sum_{i=1}^{n}\left|x_{i}^{e x}-x_{i}^{k}\right|}{\sum_{i=1}^{n} x_{i}^{e x}}$

- Standard deviation $=\frac{1}{\sqrt{n}} \sqrt{\sum_{i=1}^{n}\left(x_{i}^{k}-\bar{x}^{k}\right)^{2}}$

- Residual error $=\left\|A x^{k}-b\right\|$ in the consistent case of (1) and $\|$ $A^{T}\left(A x^{k}-b\right) \|$ in the inconsistent one

In the tests we used the box constraining function $C$ from (47) with $\alpha_{i}=$ $0, \beta_{i}=1, \forall i=1, \ldots, m$, and unitary weights $\omega_{i}, \alpha_{j}$ in (60) and (68), i.e. $\omega_{i}=1, \forall i=1, \ldots, m ; \alpha_{j}=1, \forall j=1, \ldots, n$.

Test 1: Consistent case, classical algorithms, $x^{0}=0$

We applied for the consistent problems associated to the head phantom the algorithms Cimmino (62) together with its constrained version (according to (62) and (51)) with the initial approximation $x^{0}=0$ and 500 iterations. The results presented in figures $1-2$ indicate that, in this case the constraining strategy used improve somehow the quality of the reconstructed image (1). According to the fact that the graphics in Figure 2 are almost identical, an explanation would be related to the small changes (in the positive sense) in the components of the approximations $x^{k}$, such that, also by starting with $x^{0}=0$, in 500 iterations Cimmino's algorithm together with its constrained version act almost identical.

Test 2: Consistent case, classical algorithms, $x_{i}^{0}=(-1)^{i}, i=1, \ldots, n$


Figure 1: Consistent case, $x^{0}=0,500$ iterations; left: exact, middle: Cmm, right: $\mathrm{CCmm}$.

We performed similar tests as in the above Test 1, but with the initial approximation $x_{i}^{0}=(-1)^{i}, i=1, \ldots, n$. Figure 3 indicates that the constraining strategy used, much improved in this case the quality of the reconstructed image. This aspect can be also seen in the graphics from Figure 4. 


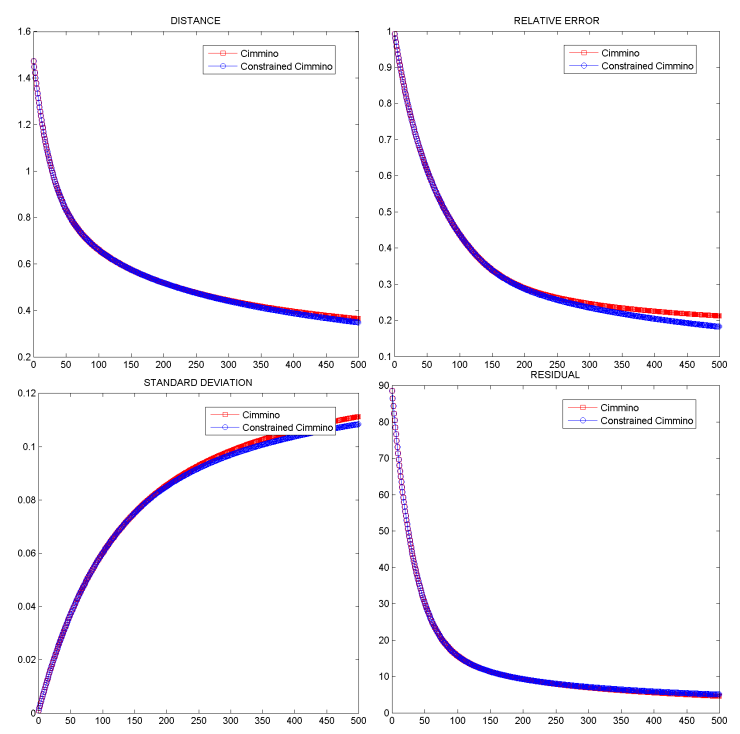

Figure 2: Consistent case, $x^{0}=0,500$ iterations; errors

Remark 8 In real reconstruction problems we will never use an initial approximation as in the above Test 2. The idea in those experiments was to show that the constraining strategy can be a very powerful tool in improving the quality of the reconstruction. The real solution for Test 1 would be an adaptive constraining strategy. Work is in progress on this subject.
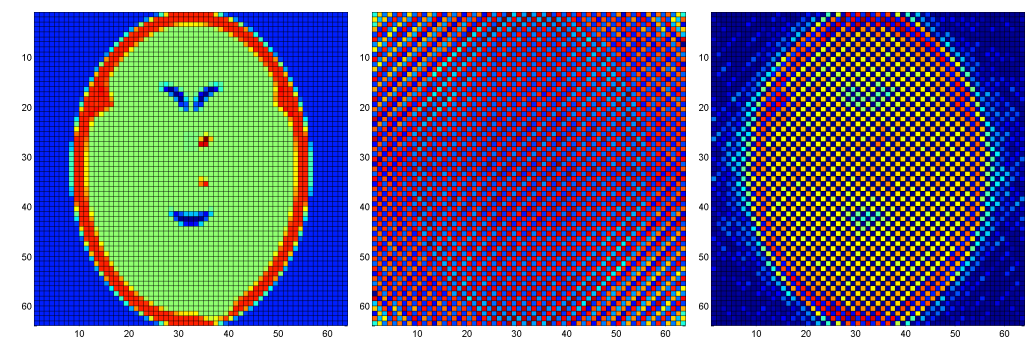

Figure 3: Consistent case, $x_{i}^{0}=(-1)^{i}, i=1, \ldots, n, 500$ iterations; left: exact, middle: Cmm, right: CCmm.

\section{Test 3: Inconsistent case, combined algorithms}

We applied for the inconsistent problems associated to both phantoms, the algorithm Cimmino (62) together with Extended Cimmino (69)-(71), 500 iterations and $x^{0}=0$. The results presented in figures 5 - 6 indicate better 

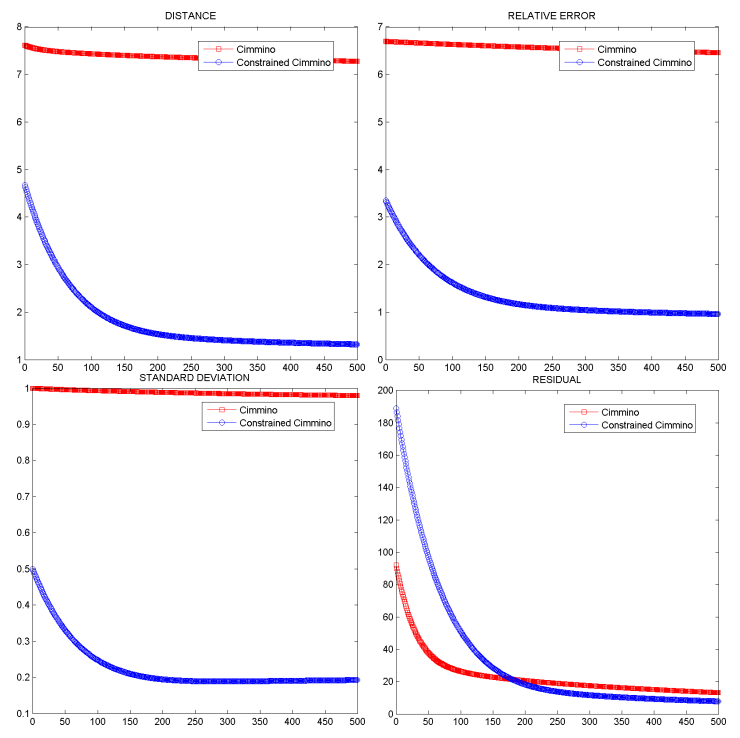

Figure 4: Consistent case, $x_{i}^{0}=(-1)^{i}, i=1, \ldots, n, 500$ iterations; errors

results for the classical version (62). Although it can be strange, this behaviour can be explained by the fact that the better theoretical properties of Extended Cimmino algorithm (69)-(71), as derived in Theorem 4, have an "asymptotic" nature, i.e. they become "visible" after an enough big number of iterations (see in this sense the similar experiments presented in Figures 7 - 8, for which 2000 iterations were used). A solution of this problem would be to improve the "right-hand side correction part" (69). Work is also in progress in this direction (see e.g. [29]).
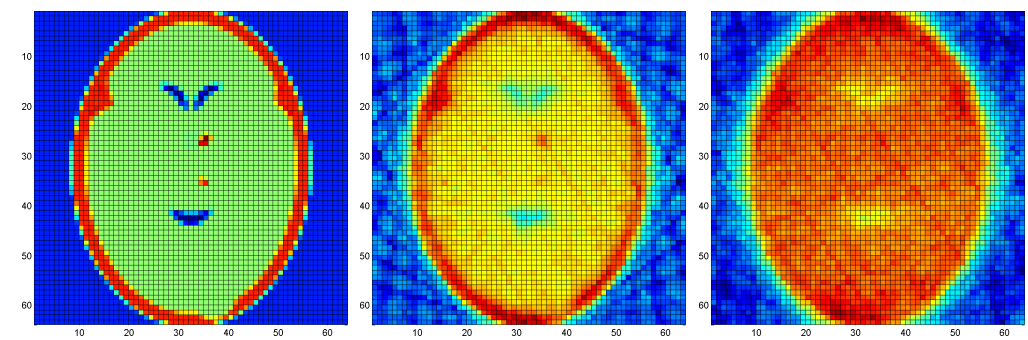

Figure 5: Inconsistent case, $x^{0}=0,500$ iterations; left: exact, middle: Cmm, right: ECmm. 


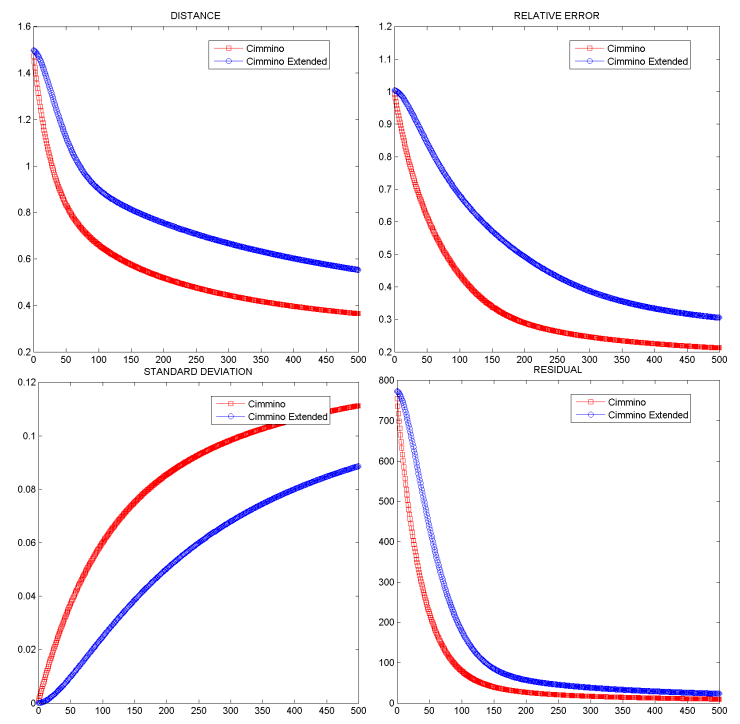

Figure 6: Inconsistent case, $x^{0}=0,500$ iterations; errors

$\mathrm{m}$

In our second set of experiments we considered the problem of 3D particle image reconstruction, which is the main step of a new technique for imaging turbulent fluids, called TomoPIV [14]. This technique is based on the instantaneous reconstruction of particle volume functions from few and simultaneous projections (2D images) of the tracer particles within the fluid. TomoPIV adopts a simple discretized model for an image-reconstruction problem, which assumes that the image consists of an array of unknowns (voxels), and sets up algebraic equations for the unknowns in terms of mea-


Figure 7: Inconsistent case, $x^{0}=0,2000$ iterations; left: exact, middle: Cmm, right: ECmm. 


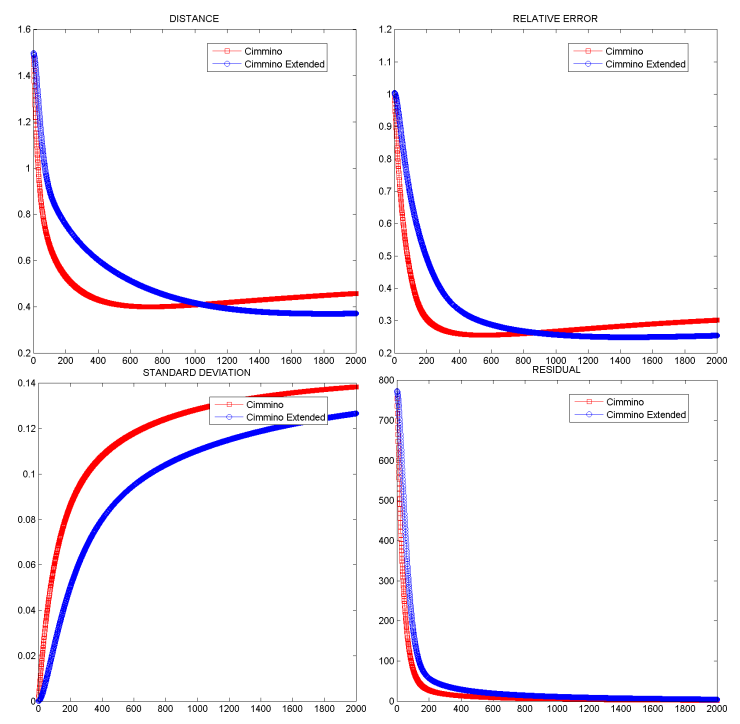

Figure 8: Inconsistent case, $x^{0}=0,2000$ iterations; errors

sured projection data. The latter are the pixel entries in the recorded 2D images. TomoPIV employs undersampling, due to the cost and complexity of the measurement apparatus, resulting in an underdetermined system of equations and thus in an ill-posed image reconstruction problem. However, this reconstruction problem can be modeled as finding the sparsest solution of an underdetermined linear system of equations. i.e.

$$
\min \|x\|_{0} \quad \text { s.t. } \mathrm{Ax}=\mathrm{b},
$$

since the original particle distribution can be well approximated with only a very small number of active voxels relative to the number of possible particle positions in a 3D domain, see e.g. [24] for details. If the original particle distribution is sparse enough and the coefficient matrix satisfies certain properties, then the indicator vector (corresponding to the active voxels) is also the unique nonnegative vector which satisfies the measurements $A x=b$ and coincides with the solution of (72).

Here, we will concentrate on a simple geometry for sampling the original particle distribution within a $64 \times 64 \times 643 \mathrm{D}$ domain from 3 orthogonal directions, compare Fig. 9. The sampling matrix $A$ will correspond to a perturbed adjacency matrix of a bipartite graph [24] were the left nodes correspond to the $64^{3}$ voxels within the volume and the right nodes to the $3 \cdot 64^{2}$ pixels within the three sampled $2 \mathrm{D}$ images, see Fig. 9 (right). 
If the number of nonzero elements in the original indicator vector $x^{*}$, i.e. $\left\|x^{*}\right\|_{0}:=\left|\left\{i \mid x_{i}^{*} \neq 0\right\}\right|$, is small enough, more precisely

$$
k:=\left\|x^{*}\right\|_{0} \leq \frac{3 d^{2}}{4 \log \frac{d}{3}} \approx 1003,
$$

then $x^{*}$ is (most probably) the unique nonnegative solution of the linear system and CGenART will converge to $x^{*}$ for the constraining function $C$ from (47). The same will hold for CGenART combined with the hard thresholding operator from (48)-(49), for carefully chosen $\alpha$. In fact, Cimmino combined with this constraining strategy is closely related to the hard thresholded Landweber iteration from [4], where this method is shown to converge to a local optimum of (72). Due to this attribute the method was only applied [4] as an prepossessing step for solution refinement obtained by other sparse approximation algorithms. However, for a carefully chosen threshold $\alpha$ the method will converge to solution of (72) provided this sparsest solution is unique. But choosing the proper $\alpha$ is an art by itself. We decided to use $\alpha=0.1$ but only after the first $0.5 k$ iterations. A combination of two constraining operators is also legitimate and turns out to be more effective in reducing the error within the same number of iterations, see Fig. 11. In fact, hard thresholding combined with box constraining has an acceleration effect reflected in a reduced number of iterations, see Fig. 10. Hence, a proper constraining strategy is an indispensable tool not only for regularization purposes (box constraining) but also for achieving computational efficiency. This issue will be addressed in further work.

Acknowledgments. We express our gratitude to Prof. Dr. Tommy Elfving and Dr. Touraj Nikazad from Linköping University Sweden for providing us the head phantom used in the numerical experiments.

\section{References}

[1] Bertero M., Boccacci P., Introduction to inverse problems in imaging, Institute of Physics Publishing, Bristol, UK, 1998.

[2] Bjork A., Elfving T. Accelerated projection methods for computing pseudoinverse solutions of systems of linear equations, B I T, 19 (1979), 145 $-163$. 

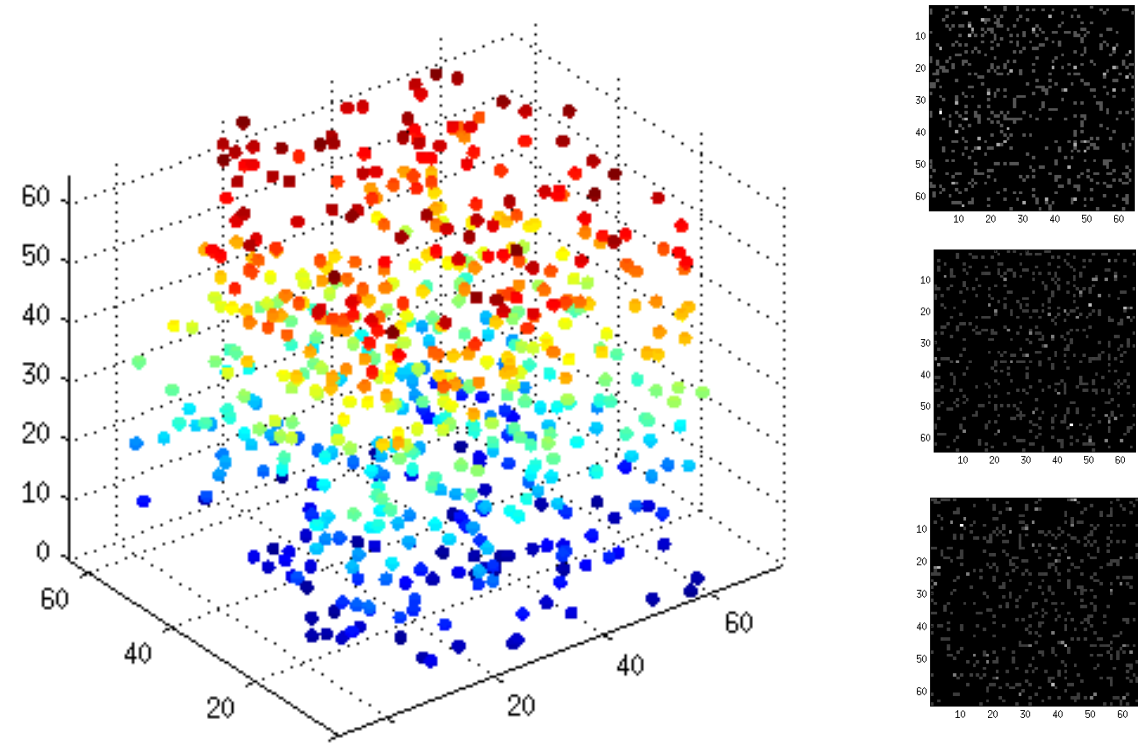

Figure 9: The original 3D particle volume function (e.g. 602 particles) has to be reconstructed from three $2 \mathrm{D}$ images $\left(64^{2}\right.$ pixel each). The indicator vector corresponding to the original particle distribution is also the unique nonnegative solution which satisfies the measurements.

[3] Bjork A., Numerical methods for least squares problems, SIAM Philadelphia, 1996.

[4] Blumensath T. and Davies M. E. Iterative thresholding for sparse approximations, J. of Fourier Anal. and Appl., 14(2008), 629 - 654.

[5] Byrne C., Block iterative interior point optimization methods for image reconstruction from limited data, Inverse problems 16(2001), 1405 1419.

[6] Censor Y., Stavros A. Z. Parallel optimization: theory, algorithms and applications, "Numer. Math. and Sci. Comp." Series, Oxford Univ. Press, New York, 1997.

[7] Censor Y., Gordon D., Gordon R. Component averaging: An efficient iterative parallel algorithm for large and sparse unstructured problems, Parallel Computing 27(2001), 777 - 808. 


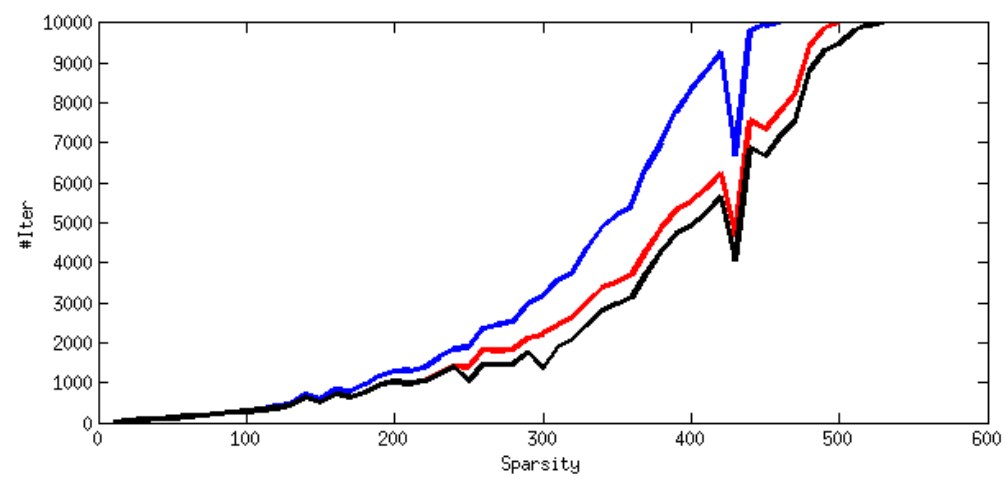

Figure 10: Reconstruction experiment for 10,20, ., 600 particles (sparsity) within the $64^{3}$ cube for Cimmino with box constraining (blue), Cimmino with hard thresholding (red) and Cimmino combined with both constraining strategies (blue). The averaged number of iterations (100 trials) increases proportional to the particle sparsity. Thresholding turns out to be more effective in attaining the stopping rule: relative error (p. 14) less then $10^{-2}$ or more then 10000 iterations.

[8] Censor Y., Elfving T. Block-iterative algorithms with diagonally scaled oblique projections for the linear feasibility problem, SIAM J. Matrix Anal. Appl., 24(1)(2002), 40 - 58.

[9] Censor Y., Elfving T., Herman G. T., Nikazad T. On diagonally relaxed orthogonal projection methods, SIAM J. Sci. Comp., 30(2007/08), 473 504.

[10] Cimmino G. Calcolo approssiomatto per le soluzioni dei sistemi di equazioni lineari, Ric. Sci. progr. tecn. econom. naz. 1 (1938), pp. 326 333.

[11] Elfving T., Block-Iterative Methods for Consistent and Inconsistent Linear Equations, Numer. Math., 35(1980), pp.1-12.

[12] Elfving T., A stationary iterative pseudoinverse algorithm, B I T, 38(2) (1998), $275-282$.

[13] Elfving T., Nikazad T., Stopping rules for Landweber-type iteration, Inverse problems, 23(2007), 1417 - 1432. 
[14] Elsinga G, Scarano F, Wieneke B, B van Oudheusden, Tomographic particle image velocimetry, Exp Fluids, 41(2006), 933 - 947.

[15] Engl H., Hanke M., Neubauer A., Regularization of inverse problems, Kluwer Academic Publishers, Boston, 2000.

[16] Herman, G. T., Image reconstruction from projections. The fundamentals of computerized tomography, Academic Press, New York, 1980.

[17] Horn R. A., Johnson C. R., Matrix analysis, Cambridge University Press, New York, 1990.

[18] Jiang M., Wang G., Convergence studies on iterative algorithms for image reconstruction, IEEE Trans. Medical Imaging, 22(2003), 569 - 579.

[19] Kaczmarz S. Angenäherte Auflösung von Systemen linearer Gleichungen, Bull. Acad. Polonaise Sci. et Lettres A (1937), 355 - 357.

[20] Koltracht I. and Lancaster P., Constraining strategies for linear iterative processes, IMA Journal of Numerical Analysis, 10(1990), 555 - 567.

[21] Natterer F., The Mathematics of Computerized Tomography, John Wiley and Sons, New York, 1986.

[22] Nicola A., Popa C., Kaczmarz Extended versus Augmented System solution in image reconstruction, Preprint CSAM 02-2009 ( http://www.univovidius.ro/math/default.aspx?cat $=$ Cercetare\&subcat $=$ Preprint\&lang $=u k)$.

[23] Petra, S., Popa C. and Schnörr C., Extended and Constrained Cimminotype Algorithms with Applications in Image Reconstruction, IWR Preprint No. 8798 (2008), University of Heidelberg. (http://www.uniheidelberg.de/archiv/8798)

[24] Petra, S. and Schnörr C., TomoPIV meets Compressed Sensing, IWR Preprint 2009, University of Heidelberg, to appear.

[25] Popa C., Least-squares solution of overdetermined inconsistent linear systems using Kaczmarz's relaxation, Intern. J. Computer Math., 55 (1995), 79 - 89 . 
[26] Popa C., Extensions of block-projection methods with relaxation parameters to inconsistent and rank-defficient least-squares problems, B I T, 38(1998), 151 - 176.

[27] Popa C., Characterization of the solutions set of inconsistent leastsquares problems by an extended Kaczmarz algorithm; Korean Journal on Comp. and Appl. Math., vol. 6(1999), 51 - 64.

[28] Popa C., Constrained Kaczmarz extended algorithm for image reconstruction, Linear Algebra and its Applications, 429(2008), 2247 - 2267.

[29] Popa C., A hybrid Kaczmarz - Conjugate Gradient algorithm, FAU Erlangen-N ürnberg, Lehrstuhl für Informatik 10 (Systemsimulation), Technical Report 07-2(2007); to appear in ...

[30] Pyle L. D., A generalised inverse $\epsilon$-algorithm for constructing intersection projection matrices with applications, Numer. Math., 10 (1967), 86 - 102.

[31] Tanabe K., Projection Method for Solving a Singular System of Linear Equations and its Applications, Numer. Math., 17(1971), 203 - 214. 

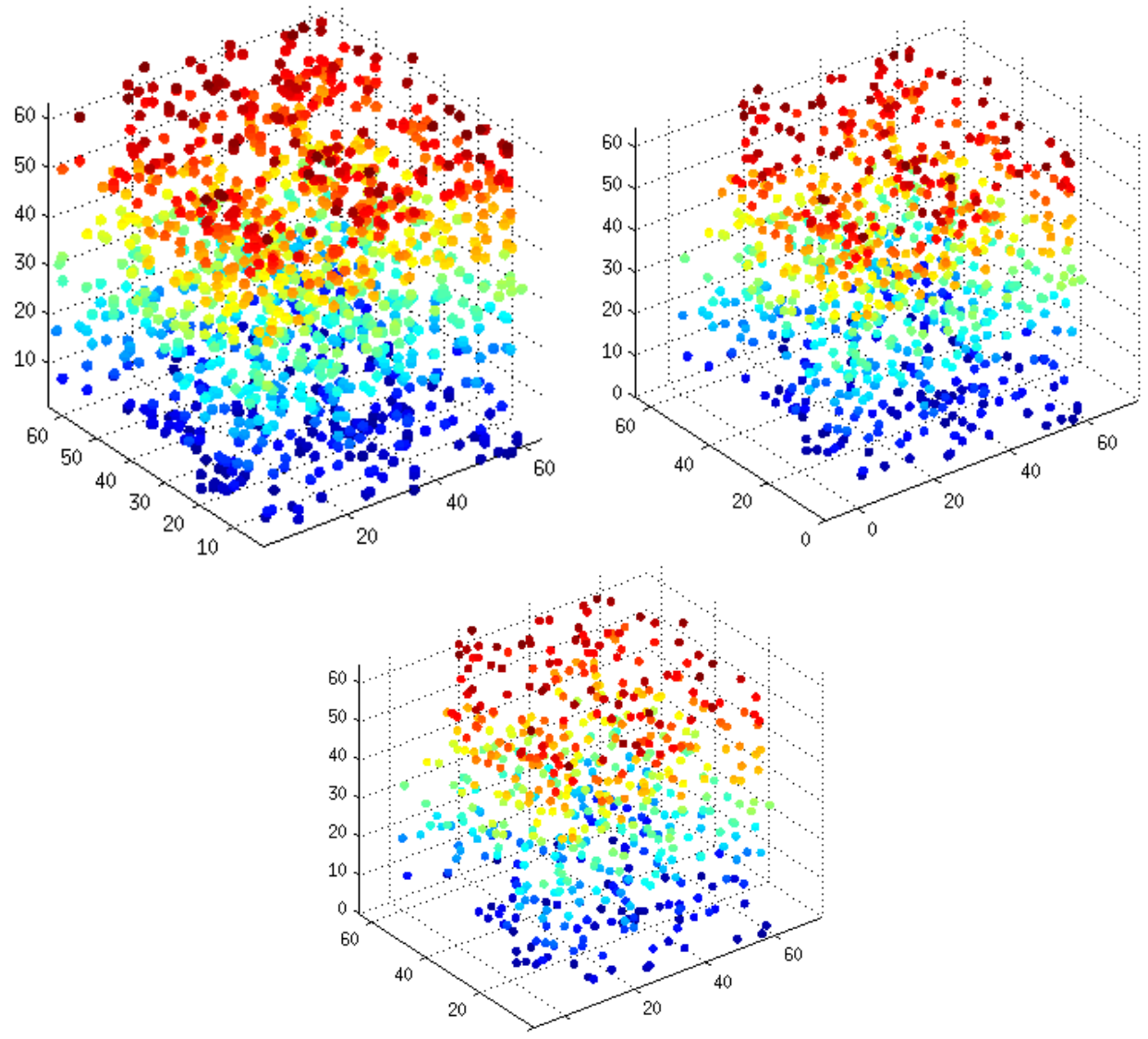

Figure 11: Reconstruction experiment for 602 particles in a $64 \times 64 \times 64$ cube from 3 orthogonal projections. In 1000 iterations of Cimmino with box constraining (47) the reconstruction (top left) contains 1246 particles exceeding a threshold 0.5. Combining box constraining with the hard thresholding operator from (48)-(49) the reconstruction improves. After 1000 iterations the reconstruction (top left) contains 827 particles - containing the original ones. The reconstruction after convergence is exact, i.e. identical with the original from Fig. 9 within the tolerance - box constraining: 18029 iterations, box constraining combined with hard thresholding: 30787 iterations (bottom). 